\title{
THE G-FUNCTIONS AS UNSYMMETRICAL FOURIER KERNELS. I
}

\author{
ROOP NARAIN
}

1. A function $K(x)$ by means of which an arbitrary function $f(x)$, subject to appropriate conditions, is capable of being represented as a repeated integral

$$
f(x)=\int_{0}^{\infty} K(u x) \int_{0}^{\infty} K(u y) f(y) d y d u
$$

has been called a Fourier kernel by Hardy and Titchmarsh [1, p. 116]. The relations (1.1) is usually written as a pair of reciprocal integral equations

$$
g(x)=\int_{0}^{\infty} K(x y) f(y) d y, \quad f(x)=\int_{0}^{\infty} K(x y) g(y) d y .
$$

(1.1) is a symmetrical formula. There are also formulae of the type

$$
f(x)=\int_{0}^{\infty} K(u x) \int_{0}^{\infty} H(u y) f(y) d y d u
$$

in which the kernels in the two integrals are different functions. This relation may also be written as a pair of unsymmetrical integral equations

$$
\begin{aligned}
& g(x)=\int_{0}^{\infty} H(x y) f(y) d y, \\
& f(x)=\int_{0}^{\infty} K(x y) g(y) d y .
\end{aligned}
$$

A simple example of the formula (1.3) is that in which

$$
K(x)=x^{1 / 2} Y_{\nu}(x) \text { and } H(x)=x^{1 / 2} H_{\nu}(x),
$$

where $Y_{\nu}(x)$ denotes the Bessel function of the first kind and $H_{\nu}(x)$ the Struve's function [4, p. 64 and p. 328].

The functions $K(x)$ and $H(x)$ have been referred to as $a$ pair of unsymmetrical Fourier kernels by various authors. The object of this paper is to obtain a new pair of kernels $K(x), H(x)$ in terms of the most general $G$-function satisfying (1.3).

The $G$-function is a sum of hypergeometric functions each of which is usually an entire function. It is written as on the left of (1.4) below and defined [2, p. 207] by the integral on the right

Received by the editors September 8, 1961 and, in revised form, October 17, 1961. 


$$
G_{p, q}^{m, n}\left(x \mid \begin{array}{l}
a_{1}, \cdots, a_{p} \\
b_{1}, \cdots, b_{q}
\end{array}\right)
$$

$$
=\frac{1}{2 \pi i} \int_{L} \frac{\prod_{j=1}^{m} \Gamma\left(b_{j}-s\right) \prod_{j=1}^{n} \Gamma\left(1-a_{j}+s\right)}{\prod_{j=m+1}^{q} \Gamma\left(1-b_{j}+s\right) \prod_{j=n+1}^{p} \Gamma\left(a_{j}-s\right)} x^{s} d s .
$$

The poles of the integrand must be simple and those of $\Gamma\left(b_{j}-s\right)$, $j=1,2, \cdots, m$, must lie on one side of the contour $L$ and those of $\Gamma\left(1-a_{j}+s\right), j=1,2, \cdots, n$, must lie on the other side.

An example of a symmetrical Fourier kernel in terms of $G$-function has been given by the author [3, p. 298] earlier as

$$
x^{1 / 2} G_{2 p, 2 q}^{q, p}\left(\frac{1}{4} x^{2} \mid \begin{array}{l}
a_{1}, \cdots, a_{p},-a_{1}, \cdots,-a_{p} \\
b_{1}, \cdots, b_{q},-b_{1}, \cdots,-b_{q}
\end{array}\right) .
$$

It was also pointed out that

$$
2 \beta x^{1 / 2} G_{2 p, 2 q}^{q, p}\left(\beta^{2} x^{2} \mid \begin{array}{l}
a_{1}, \cdots, a_{p},-a_{1}, \cdots,-a_{p} \\
b_{1}, \cdots, b_{q},-b_{1}, \cdots,-b_{q}
\end{array}\right)
$$

and

$$
2 \beta \gamma x^{\gamma-1 / 2} G_{2 p, 2 q}^{q, p}\left(\beta^{2} x^{2 \gamma} \mid \begin{array}{l}
a_{1}, \cdots, a_{p},-a_{1}, \cdots,-a_{p} \\
b_{1}, \cdots, b_{q},-b_{1}, \cdots,-b_{q}
\end{array}\right)
$$

where $\beta$ and $\gamma$ are real constants, are also Fourier kernels.

2. Let $\Omega(s)$ and $\mathfrak{W}(s)$ be the Mellin transforms of $K(x)$ and $H(x)$ respectively, i.e.

$$
\Re(s)=\int_{0}^{\infty} x^{s-1} K(x) d x, \quad \mathfrak{g}(s)=\int_{0}^{\infty} x^{s-1} H(x) d x
$$

then for the validity of $(1.3) \Re(s)$ and $\mathfrak{S}(s)$ must satisfy $[1$, p. 118] the functional relation

$$
\Re(s) \mathfrak{S}(1-s)=1,
$$

and $K(x), H(x)$ and $f(x)$ must satisfy certain convergence conditions.

Consider the functions

$$
K(x)=A x^{1 / 2} G_{p+q, m+n}^{m, p}\left(\frac{1}{4} x^{2} \mid \begin{array}{l}
a_{1}, \cdots, a_{p}, b_{1}, \cdots, b_{q} \\
c_{1}, \cdots, c_{m}, d_{1}, \cdots, d_{n}
\end{array}\right)
$$

and 


$$
H(x)=B x^{1 / 2} G_{p+q, m+n}^{n, q}\left(\frac{1}{4} x^{2} \mid \begin{array}{l}
\beta_{1}, \cdots, \beta_{q}, \alpha_{1}, \cdots, \alpha_{p} \\
\delta_{1}, \cdots, \delta_{n}, \gamma_{1}, \cdots, \gamma_{m}
\end{array}\right)
$$

where $A$ and $B$ are constants. The Mellin transforms of $K(x)$ is

$$
\begin{aligned}
\Re(s) & =A \int_{0}^{\infty} x^{s-1 / 2} G_{p+q, m+n}^{m, p}\left(\frac{1}{4} x^{2} \mid \begin{array}{l}
a_{1}, \cdots, a_{p}, b_{1}, \cdots, b_{q} \\
c_{1}, \cdots, c_{m}, d_{1}, \cdots, d_{n}
\end{array}\right) d x \\
& =2^{s-1 / 2} A \int_{0}^{\infty} y^{(s / 2+1 / 4)-1} G_{p+q, m+n}^{m, p}\left(y \mid \begin{array}{l}
a_{1}, \cdots, a_{p}, b_{1}, \cdots, b_{q} \\
c_{1}, \cdots, c_{m}, d_{1}, \cdots, d_{n}
\end{array}\right) d y .
\end{aligned}
$$

Evaluating this by a known integral [5, p. 337], we get

$$
\begin{aligned}
& \mathbb{R}(s)=2^{,-1 / 2} A \frac{\prod_{j=1}^{m} \Gamma\left(c_{j}+1 / 4+s / 2\right) \prod_{j=1}^{p} \Gamma\left(3 / 4-a_{j}-s / 2\right)}{\prod_{j=1}^{n} \Gamma\left(3 / 4-d_{j}-s / 2\right) \prod_{j=1}^{q} \Gamma\left(1 / 4+b_{j}+s / 2\right)}, \\
& -\min _{1 \leqq j \leqq m} \operatorname{Re}\left(c_{j}\right)<\operatorname{Re}(s / 2+1 / 4)<1-\max _{1 \leqq j \leqq p} \operatorname{Re}\left(a_{j}\right) \text {, } \\
& p+q<m+n \text {. }
\end{aligned}
$$

Similarly,

$$
\begin{gathered}
\mathfrak{S}(s)=2,-1 / 2 B \frac{\prod_{j=1}^{n} \Gamma\left(1 / 4+\delta_{j}+s / 2\right) \prod_{j=1}^{q} \Gamma\left(3 / 4-\beta_{j}-s / 2\right)}{\prod_{j=1}^{m} \Gamma\left(3 / 4-\gamma_{j}-s / 2\right) \prod_{j=1}^{p} \Gamma\left(1 / 4+\alpha_{j}+s / 2\right)}, \\
-\min _{1 \leqq 1 \leqq n} \operatorname{Re}\left(\delta_{j}\right)<\operatorname{Re}(s / 2+1 / 4)<1-\max _{1 \leqq 1 \leqq q} \operatorname{Re}\left(\beta_{j}\right), \\
p+q<m+n .
\end{gathered}
$$

These satisfy the functional relation (2.2) if

$$
\begin{aligned}
2-1 / 2 A & \frac{\prod_{j=1}^{m} \Gamma\left(1 / 4+c_{j}+s / 2\right) \prod_{j=1}^{p} \Gamma\left(3 / 4-a_{j}-s / 2\right)}{\prod_{j=1}^{n} \Gamma\left(3 / 4-d_{j}-s / 2\right) \prod_{j=1}^{q} \Gamma\left(1 / 4+b_{j}+s / 2\right)} \\
& \times 2^{1 / 2-s} B \frac{\prod_{j=1}^{n} \Gamma\left(3 / 4+\delta_{j}-s / 2\right) \prod_{j=1}^{q} \Gamma\left(1 / 4-\beta_{j}+s / 2\right)}{\prod_{j=1}^{m} \Gamma\left(1 / 4-\gamma_{j}+s / 2\right) \prod_{j=1}^{p} \Gamma\left(3 / 4+\alpha_{j}-s / 2\right)}=1 .
\end{aligned}
$$


This relation admits of a solution if

$$
\begin{aligned}
A B & =1, & & \\
a_{j}+\alpha_{j} & =0, & & j=1, \cdots, p, \\
b_{j}+\beta_{j} & =0, & & j=1, \cdots, q, \\
c_{j}+\gamma_{j} & =0, & j & =1, \cdots, m, \\
d_{j}+\delta_{j} & =0, & j & =1, \cdots, n .
\end{aligned}
$$

Hence we have

$$
\begin{aligned}
& K(x)=A x^{1 / 2} G_{p+q, m+n}^{m, p}\left((1 / 4) x^{2} \mid \begin{array}{l}
a_{1}, \cdots, a_{p}, b_{1}, \cdots, b_{q} \\
c_{1}, \cdots, c_{m}, d_{1}, \cdots, d_{n}
\end{array}\right), \quad \text { and } \\
& (2.3) \\
& H(x)=A^{-1} x^{1 / 2} G_{p+q, m+n}^{n, q}\left((1 / 4) x^{2} \mid \begin{array}{l}
-b_{1}, \cdots,-b_{q},-a_{1}, \cdots,-a_{p} \\
-d_{1}, \cdots,-d_{n},-c_{1}, \cdots,-c_{m}
\end{array}\right)
\end{aligned}
$$

as a pair of kernel functions giving rise (formally) to the unsymmetrical formula (1.3). The formulation of convergence conditions with which the formal analysis is justifiable is a matter which will be discussed in a subsequent paper [6]. The importance of these functions is due to their general form from which many known as well as unknown kernels can be deduced as particular cases.

2.1. It is easy to show that if $K(x)$ and $H(x)$ form a pair of unsymmetrical Fourier kernels so also are the pairs

and

$$
\sqrt{ } \beta K(\beta x), \quad \sqrt{ } \beta H(\beta x)
$$

$$
\gamma x^{1 / 2(\gamma-1)} K\left(x^{\gamma}\right), \quad \gamma x^{1 / 2(\gamma-1)} H\left(x^{\gamma}\right),
$$

where $\beta$ and $\gamma$ are real constants.

Using this we find that

$$
\begin{aligned}
& 2 \beta A x^{1 / 2} G_{p+q, m+n}^{m, p}\left(\beta^{2} x^{2} \mid \begin{array}{l}
a_{1}, \cdots, a_{p}, b_{1}, \cdots, b_{q} \\
c_{1}, \cdots, c_{m}, d_{1}, \cdots, d_{n}
\end{array}\right), \\
& 2 \beta A^{-1} x^{1 / 2} G_{p+q, m+n}^{n, q}\left(\beta^{2} x^{2} \mid \begin{array}{l}
-b_{1}, \cdots,-b_{q},-a_{1}, \cdots,-a_{p} \\
-d_{1}, \cdots,-d_{n},-c_{1}, \cdots,-c_{m}
\end{array}\right)
\end{aligned}
$$

form a pair of unsymmetrical Fourier kernels and so also

$$
\begin{aligned}
& 2 \beta \gamma A x^{\gamma-1 / 2} G_{p+q, m+n}^{m, p}\left(\beta^{2} x^{2 \gamma} \mid \begin{array}{l}
a_{1}, \cdots, a_{p}, b_{1}, \cdots, b_{q} \\
c_{1}, \cdots, c_{m}, d_{1}, \cdots, d_{n}
\end{array}\right), \\
& 2 \beta \gamma A^{-1} x^{\gamma-1 / 2} G_{p+q, m+n}^{n, q}\left(\beta^{2} x^{2 \gamma} \mid \begin{array}{l}
-b_{1}, \cdots,-b_{q},-a_{1}, \cdots,-a_{p} \\
-d_{1}, \cdots,-d_{n},-c_{1}, \cdots,-c_{m}
\end{array}\right) .
\end{aligned}
$$


3. Particular cases. Giving suitable values to the parameters in (2.3), (2.4), (2.5), we can deduce as particular cases a number of kernels determined earlier. ${ }^{1}$ We give here a few examples of them. The notations for the various transcendental functions occurring below are the same as used by Watson [4, p. 789].

(a) The functions

$$
K(x)=x^{1 / 2} G_{13}^{11}\left((1 / 4) x^{2} \begin{array}{l}
1 / 2+\nu / 2 \\
1 / 2+\nu / 2, \nu / 2,-\nu / 2
\end{array}\right) \equiv x^{1 / 2} H_{\nu}(x),
$$

and

$$
H(x)=x^{1 / 2} G_{13}^{20}\left((1 / 4) x^{2} \mid \begin{array}{l}
-1 / 2-\nu / 2 \\
-\nu / 2, \nu / 2,-1 / 2-\nu / 2
\end{array}\right) \equiv x^{1 / 2} Y_{\nu}(x)
$$

are the unsymmetrical Fourier kernels obtained by Titchmarsh $[7$, p. xxxiv].

(b) Let $\phi=\alpha_{1}-\rho_{1}-\rho_{2}+1 / 2$ and let ${ }_{1} f_{2}$ denote the hypergeometric function defined by Fox [8, p. 401]. The functions

$$
\begin{aligned}
K(x) & =x^{1 / 2}(x / 2)^{3 / 2-\phi} G_{13}^{11}\left((1 / 4) x^{2} \mid \begin{array}{c}
-\alpha_{1} \\
-1,-\rho_{1},-\rho_{2}
\end{array}\right) \\
& \equiv x^{1 / 2}(x / 2)^{-\phi-1 / 2}{ }_{1} f_{2}\left\{\begin{array}{c}
\alpha_{1} \\
\rho_{1}, \rho_{2}
\end{array} ;-(1 / 4) x^{2}\right\}
\end{aligned}
$$

and

$$
\begin{aligned}
H(x)= & x^{1 / 2}(x / 2)^{\phi-3 / 2} G_{13}^{20}\left((1 / 4) x^{2} \mid \begin{array}{c}
\alpha_{1} \\
\rho_{1}, \rho_{2}, 1
\end{array}\right) \\
\equiv & x^{1 / 2} \sum_{\rho_{1}, \rho_{2}} \frac{\operatorname{Sin}\left(\alpha_{1}-\rho_{1}\right) \pi}{\operatorname{Sin}\left(\rho_{2}-\rho_{1}\right) \pi}((1 / 2) x)^{2 \rho_{1}+\phi-3 / 2} \\
& { }_{1} f_{2}\left\{\begin{array}{c}
1-\alpha_{1}+\rho_{1} \\
1-\rho_{2}+\rho_{1}, \rho_{1}
\end{array} ;-(1 / 4) x^{2}\right\}
\end{aligned}
$$

form $^{2}$ the special case $p=1$ of Fox's Theorem 1 [8, p. 402].

${ }^{1}$ Reference of the known cases has been indicated as far as it has been possible.

2 The symbol $\Sigma_{\rho_{1}, \rho_{2}}$ denotes that to the expression following it a similar expression with $\rho_{1}, \rho_{2}$ interchanged is to be added. 
With $\alpha_{1}=1, \rho_{1}=\alpha+1, \rho_{2}=\nu+\alpha+1$, we have

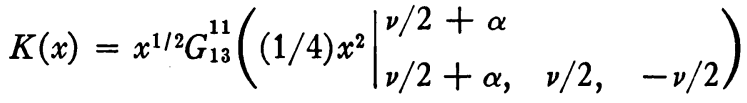

$$
\begin{aligned}
& \equiv x^{1 / 2}(x / 2)^{\nu+2 \alpha_{1} f_{2}}\left\{\begin{array}{c}
1 \\
1+\alpha, 1+\alpha+\nu
\end{array} ;-(1 / 4) x^{2}\right\}
\end{aligned}
$$

and

$$
\begin{aligned}
H(x) & =x^{1 / 2} G_{13}^{20}\left((1 / 4) x^{2} \mid \begin{array}{l}
-\nu / 2-\alpha \\
-\nu / 2, \quad \nu / 2, \quad-\nu / 2-\alpha
\end{array}\right) \\
& \equiv x^{1 / 2}\left[\operatorname{Cos} \alpha \pi J_{\nu}(x)+\operatorname{Sin} \alpha \pi Y_{\nu}(x)\right],
\end{aligned}
$$

a result due to Hardy [9, p. 1xii].

When $\alpha=0$, these formulae reduce to

$$
K(x)=H(x)=x^{1 / 2} J_{\nu}(x),
$$

the famous Hankel's formula and when $\alpha=1 / 2$, they reduce to the case (a).

(c) Let $\phi=\alpha_{1}+\alpha_{2}-\rho_{1}-\rho_{2}-\rho_{3}+1 / 2$. The functions

$$
\begin{aligned}
& K(x)=x^{1 / 2}(x / 2)^{3 / 2-\phi} G_{24}^{12}\left((1 / 4) x^{2} \mid \begin{array}{llll}
-\alpha_{1}, & -\alpha_{2} & \\
-1, & -\rho_{1}, & -\rho_{2}, & -\rho_{3}
\end{array}\right) \\
& \equiv x^{1 / 2}(x / 2)^{-\phi-1 / 2}{ }_{2} f_{3}\left\{\begin{array}{c}
\alpha_{1}, \alpha_{2} \\
\rho_{1}, \rho_{2}, \rho_{3}
\end{array} ;-(1 / 4) x^{2}\right\}
\end{aligned}
$$

and

$$
\begin{aligned}
& H(x)=x^{1 / 2}(x / 2)^{\phi-3 / 2} G_{24}^{30}\left((1 / 4) x^{2} \mid \begin{array}{llll}
\alpha_{1}, & \alpha_{2} & \\
\rho_{1}, & \rho_{2}, & \rho_{3}, & 1
\end{array}\right) \\
& \equiv x^{1 / 2}(x / 2)^{\phi-3 / 2} \sum_{\rho_{1}, \rho_{2}, \rho_{2}} \frac{\operatorname{Sin}\left(\alpha_{1}-\rho_{1}\right) \pi \operatorname{Sin}\left(\alpha_{2}-\rho_{1}\right) \pi}{\operatorname{Sin}\left(\rho_{2}-\rho_{1}\right) \pi \operatorname{Sin}\left(\rho_{3}-\rho_{1}\right) \pi}((1 / 2) x)^{2 \rho_{1}} \\
& { }_{2} f_{3}\left\{\begin{array}{c}
1-\alpha_{1}+\rho_{1}, 1-\alpha_{2}+\rho_{1} \\
1-\rho_{2}+\rho_{1}, 1-\rho_{3}+\rho_{1}, \rho_{1}
\end{array} ;-\frac{x^{2}}{4}\right\},
\end{aligned}
$$

form ${ }^{3}$ the special case $p=2$ of Fox's Theorem 1 [8, p. 402].

With $\alpha_{1}=1, \alpha_{2}=\nu+a+3 / 2, \rho_{1}=a+1, \rho_{2}=\nu+a+1, \rho_{3}=2 \nu+a+1$,

3 The symbol $\Sigma \rho_{1}, \rho_{2}, \rho_{3}$ denotes the sum of three terms in which each term is obtained from the preceding one by cyclically interchanging $\rho_{1}, \rho_{2}, \rho_{3}$. 
we have $[8$, p. 450$]$

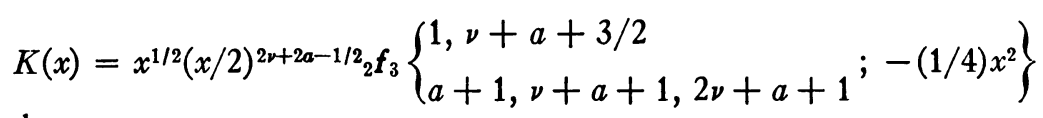

and

$$
\begin{aligned}
& H(x)=-\frac{x^{1 / 2}}{2}((1 / 2) \pi x)^{1 / 2} \\
& \text { - }\left[\operatorname{Sin} a \pi J_{-\nu}^{2}(x / 2)-2 \operatorname{Sin}(\nu+a) \pi J_{-\nu}(x / 2) J_{\nu}(x / 2)\right. \\
& \left.+\operatorname{Sin}(2 \nu+a) \pi J_{\nu}^{2}((1 / 2) x)\right] \operatorname{Cosec}^{2} \nu \pi \text {. }
\end{aligned}
$$

(d) Take $\phi=1 / 2+\sum_{r=1}^{p} \alpha_{r}-\sum_{s=1}^{p+1} \rho_{s .}$ The functions

$$
\begin{aligned}
& K(x)=x^{1 / 2}(x / 2)^{3 / 2-\phi} G_{p, p+2}^{1, p}\left((1 / 4) x^{2} \mid \begin{array}{l}
-\alpha_{1}, \cdots,-\alpha_{p} \\
-1,-\rho_{1}, \cdots,-\rho_{p+1}
\end{array}\right), \\
& H(x)=x^{1 / 2}(x / 2)^{\phi-3 / 2} G_{p, p+2}^{p+1,0}\left((1 / 4) x^{2} \mid \begin{array}{l|l}
\alpha_{1}, \cdots, \alpha_{p} \\
\rho_{1}, \cdots, \rho_{p+1}, 1
\end{array}\right)
\end{aligned}
$$

form a pair of unsymmetrical Fourier kernels of which one is a hypergeometric function of the type ${ }_{p} \boldsymbol{f}_{p+1}$ while the other is a combination of $p+1$ hypergeometric functions of the same type.

(e) Let $\phi=\alpha_{1}+\alpha_{2}-\rho_{1}-\rho_{2}-\rho_{3}+1 / 2$. The functions

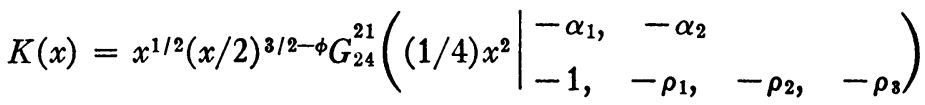

$$
\begin{aligned}
& \equiv x^{1 / 2}(x / 2)^{-\phi-1 / 2}\left[\frac{\operatorname{Sin} \alpha_{2} \pi}{\operatorname{Sin} \rho_{1} \pi}{ }_{2} f_{3}\left\{\begin{array}{c}
\alpha_{1}, \alpha_{2} \\
\rho_{1}, \rho_{2}, \rho_{3}
\end{array} ;-(1 / 4) x^{2}\right\}\right. \\
& -\frac{\operatorname{Sin}\left(\rho_{1}-\alpha_{2}\right) \pi}{\operatorname{Sin} \rho_{1} \pi}(x / 2)^{2-2 \rho_{1}} \\
& \left.{ }_{2} f_{3}\left\{\begin{array}{c}
1-\rho_{1}+\alpha_{1}, 1-\rho_{1}+\alpha_{2} \\
2-\rho_{1}, 1-\rho_{1}+\rho_{2}, 1-\rho_{1}+\rho_{3}
\end{array} ;-\frac{x^{2}}{4}\right\}\right]
\end{aligned}
$$

and

$$
\begin{aligned}
& H(x)=x^{1 / 2}(x / 2)^{\phi-3 / 2} G_{24}^{21}\left((1 / 4) x^{2} \mid \begin{array}{llll}
\alpha_{2}, & \alpha_{1} & \\
\rho_{3}, & \rho_{2}, & \rho_{1}, & 1
\end{array}\right) \\
& \equiv x^{1 / 2} \sum_{\rho_{2}, \rho_{3}} \frac{\operatorname{Sin}\left(\alpha_{1}-\rho_{2}\right) \pi}{\operatorname{Sin}\left(\rho_{3}-\rho_{2}\right) \pi}(x / 2)^{2 \rho_{2}+\phi-3 / 2} \\
& { }_{2} f_{3}\left\{\begin{array}{c}
1+\rho_{2}-\alpha_{1}, 1+\rho_{2}-\alpha_{2} \\
1+\rho_{2}-\rho_{3}, 1+\rho_{2}-\rho_{1}, \rho_{2}
\end{array} ;-(1 / 4) x^{2}\right\}
\end{aligned}
$$


form a pair of unsymmetrical Fourier kernels.

(f) The functions

$$
\begin{aligned}
K(x) & =(1 / 4) x^{3 / 2} G_{04}^{20}\left((x / 4)^{4} \mid-\frac{3}{8}, \frac{3}{8}, \frac{1}{8},-\frac{1}{8}\right) \\
& \equiv \pi^{-1 / 2}\left[\operatorname{Cos} x+\operatorname{Sin} x+e^{-x}\right]
\end{aligned}
$$

and

$$
\begin{aligned}
H(x) & =(1 / 4) x^{8 / 2} G_{04}^{20}\left((x / 4)^{4} \mid-\frac{1}{8}, \frac{1}{8}, \frac{3}{8},-\frac{3}{8}\right) \\
& \equiv \pi^{-1 / 2}\left[\operatorname{Cos} x+\operatorname{Sin} x-e^{-x}\right]
\end{aligned}
$$

are the special case of (2.5) with $\gamma=2$ and $\beta=2^{-4}$. These unsymmetrical Fourier kernels were obtained by Guinand [10, p. 192].

4. In this section some symmetrical Fourier kernels are mentioned which are particular cases of (1.6) or (1.7).

(a) With $\gamma=2$ and $\beta=2^{-4}$, the following particular cases of (1.7) may be noted.

$$
\begin{aligned}
(1 / 4) x^{3 / 2} G_{04}^{20}\left((x / 4)^{4} \mid-\frac{3}{8},-\frac{1}{8}, \frac{3}{8}, \frac{1}{8}\right) & \equiv \pi^{-1 / 2}\left[e^{-x}+\operatorname{Cos} x-\operatorname{Sin} x\right] \\
(1 / 4) x^{3 / 2} G_{04}^{20}\left((x / 4)^{4} \mid \frac{1}{8}, \frac{3}{8},\right. & \left.-\frac{1}{8},-\frac{3}{8}\right) \\
& \equiv \pi^{-1 / 2}\left[e^{-x}-\operatorname{Cos} x+\operatorname{Sin} x\right] .
\end{aligned}
$$

These kernels were given by Guinand [10, p. 192].

(b) With $\beta=2^{-n}$, where $n$ is a positive integer, a special case of (1.6) is

$2^{1-n} x^{1 / 2} G_{0,2 n}^{n, 0}\left(\frac{x^{2}}{2^{2 n}} \mid \frac{\mu_{1}}{2}, \cdots, \frac{\mu_{n}}{2},-\frac{\mu_{1}}{2}, \cdots,-\frac{\mu_{n}}{2}\right) \equiv \varpi_{\mu_{1}}, \cdots, \mu_{n}(x)$

where $\varpi_{\mu_{1}}, \ldots, \mu_{n}(x)$ is the kernel defined by Bhatnagar $[11$, p. 43$]$.

When $n=2$, we have

$$
(1 / 2) x^{1 / 2} G_{04}^{20}\left(\frac{x^{2}}{16} \mid \frac{\mu}{2}, \frac{\nu}{2},-\frac{\mu}{2},-\frac{\nu}{2}\right) \equiv \varpi_{\mu, \nu}(x),
$$

where $\varpi_{\mu, \nu}(x)$ is the kernel given by Watson $[12$, p. 308].

When $n=1$, this reduces to the Hankel's kernel $x^{1 / 2} J_{\nu}(x)$.

(c) Recently Everitt [13, p. 271] has given a generalization of 
Bessel functions. The new functions, denoted by $J_{\nu, k}(x)$, satisfy certain differential equations of even order, greater than two, and have properties similar to the Bessel functions. Looking at the Mellin transform [13, p. 275] of $x^{1 / 2} J_{\nu, k}(x)$, it has been possible to express it as

$$
\begin{aligned}
x^{1 / 2} J_{\nu, k}(x) & =(2 k)^{1 / 2}\left(\frac{x}{2 k}\right)^{k-1 / 2} G_{0,2 k}^{k, 0}\left(\left(\frac{x}{2 k}\right)^{2 k} \mid \frac{\nu}{2 k}, \frac{\nu+1}{2 k}, \ldots,\right. \\
& \left.\frac{\nu+k-1}{2 k}, \frac{\nu-k+1}{2 k}, \ldots, \frac{\nu-2}{2 k}, \frac{\nu-1}{2 k}, \frac{1-2 k}{2 k} \nu\right),
\end{aligned}
$$

$k$ being a positive integer.

When $k=1$,

$$
x^{1 / 2} J_{\nu, 1}(x)=x^{1 / 2} G_{02}^{10}\left((1 / 2) x^{2} \mid \frac{\nu}{2},-\frac{\nu}{2}\right) \equiv x^{1 / 2} J_{\nu}(x), \quad \nu>-1 / 2 .
$$

When $k>1, x^{1 / 2} J_{\nu, k}(x)$ is of the form (1.6) only when $\nu=0$ or $\nu=1 / 2$. With $\nu=0$ and $\nu=1 / 2$, we have Everitt's kernels [13, p. 271]

$$
\begin{aligned}
x^{1 / 2} J_{0, k}(x) & =(2 k)^{1 / 2}\left(\frac{x}{2 k}\right)^{k-1 / 2} \\
\cdot G_{0,2 k}^{k, 0} & \left(\left(\frac{x}{2 k}\right)^{2 k} \mid 0, \frac{1}{2 k}, \ldots, \frac{k-1}{2 k}, 0, \frac{-1}{2 k}, \ldots,-\frac{k-1}{2 k}\right)
\end{aligned}
$$

and

$$
\begin{aligned}
& x^{1 / 2} J_{1 / 2, k}(x)=(2 k)^{1 / 2}\left(\frac{x}{2 k}\right)^{k-1 / 2} \\
& \cdot G_{0,2 k}^{k, 0}\left(\left(\frac{x}{2 k}\right)^{2 k} \mid \frac{1}{4 k}, \frac{3}{4 k}, \ldots, \frac{2 k-1}{4 k}, \frac{-1}{4 k}, \frac{-3}{4 k}, \ldots,-\frac{2 k-1}{4 k}\right) .
\end{aligned}
$$

Note that these are the special cases $\gamma=k, \beta=(2 k)^{-k}$ of (1.7).

\section{REFERENCES}

1. G. H. Hardy and E. C. Titchmarsh, A class of Fourier kernels, Proc. London Math. Soc. Ser. II 35 (1933), 116-155.

2. Bateman manuscript project, Higher transcedental functions, 1 (1953).

3. R. Narain, A Fourier kernel, Math. Z. 70 (1959), 297-299.

4. G. N. Watson, Theory of Bessel functions, (1944).

5. Bateman manuscript project, Tables of integral transforms, 1 (1954).

6. R. Narain, The G-functions as unsymmetrical Fourier kernels. II, III, Proc. Amer. Math. Soc. (to appear). 
7. E. C. Titchmarsh, $A$ pair of inversion formulae, Proc. London Math. Soc. Ser. II 22 (1923).

8. C. Fox, A generalization of the Fourier-Bessel integral, Proc. London Math. Soc. Ser. II 29 (1929), 401-452.

9. G. H. Hardy, Some formulae in the theory of Bessel functions, Proc. London Math. Soc. Ser. II 23 (1924), lxi-lxiii.

10. A. P. Guinand, A class of Fourier kernels, Quart. J. Math. Oxford Ser. II 1 (1950), 191-193.

11. K. P. Bhatnagar, Two theorems on self-reciprocal functions and a new transform. Bull. Calcutta Math. Soc. 45 (1953), 109-112.

12. G. N. Watson, Some self-reciprocal functions, Quart. J. Math. Oxford Ser. I 2 (1931), 298-309.

13. W. N. Everitt, On a generalization of Bessel functions and a resulting class of Fourier kernels, Quart. J. Math., Oxford Ser. II 10 (1959), 270-279.

Punjab University, Chandigarh, India and WASHINGTON UNIVERSITY 